\title{
Nutrition and Metabolic Insights
}

\section{Effect of the DASH Diet on Pre- and Stage 1 Hypertensive Individuals in a Free-Living Environment}

\author{
Joan Malloy-McFall, Jacob E. Barkley, Karen Lowry Gordon, Nancy Burzminski and Ellen L. Glickman \\ The School of Health Sciences, Kent State University, Kent OH, USA. Email: jmcfall@kent.edu
}

\begin{abstract}
Background: Dietary Approaches to Stop Hypertension (DASH) has been shown to successfully reduce systolic (SBP) and diastolic blood pressure (DBP) when evaluated in clinically controlled environments but there is a lack of information regarding the efficacy of the original DASH diet when it is applied in a free-living environment.

Purpose: To provide descriptive data as to the changes in blood pressure individuals could expect to achieve when following the DASH diet in a free-living environment for 4-weeks with no additional behavioral modifications.

Methods: Twenty, pre- and stage 1 hypertensive participants were randomly split into 2 groups; DASH (males $\mathrm{N}=5$, females $\mathrm{N}=5$, age $=38.5 \pm 10.8$ ) and control (males $\mathrm{N}=7$, females $\mathrm{N}=3$, age $=38.1 \pm 11.1$ ). The DASH group was instructed on how to follow the DASH diet on their own for 4-weeks while the control group continued their normal diet. SBP, DBP, body weight, 3-day food diaries and physical activity recall questionnaire data were collected pre and post intervention using a traditional person-to-person instructional technique.

Results: Two-way ANOVA demonstrated that there was a significant group (DASH, control) by time (pre, post) interaction for SBP $(P=0.003)$ and no significant effects for DBP. The interaction was due to a significant reduction $(P<0.001)$ in SBP in the DASH group (pre: $141.3 \pm 11.3 \mathrm{mmHg}$ vs. post: $130.7 \pm 9.1 \mathrm{mmHg}$ ) over the course of the intervention with no change in SBP in the control group (pre: $133.5 \pm 6.6 \mathrm{mmHg}$ vs. post: $131.9 \pm 8.9 \mathrm{mmHg}$ ). Pearson's correlation analyses revealed that changes in potential moderators of blood pressure including body weight, BMI, sodium intake and total kilocalories were each not associated with changes in SBP ( $\mathrm{r} \leq 0.14, P \geq 0.5)$ or DBP ( $\mathrm{r} \leq 0.10, P \geq 0.6)$ pre- to post-treatment. Chi-square demonstrated no significant differences in the number of participants per group ( $\mathrm{n}=4 \mathrm{DASH}, \mathrm{n}=1$ control) who indicated increasing physical activity during the intervention.

Conclusion: DASH diet followed in a free-living environment significantly reduced SBP but not DBP. However, the changes in SBP and DBP were very similar to those noted in controlled clinical feeding evaluations of the DASH diet. Presently, none of the potential moderators of blood pressure that were assessed were independently associated with the observed changes in blood pressure which may be due to our small sample size or the possibility that it is the combined change in multiple factors that lead to reductions in blood pressure when following the DASH diet.
\end{abstract}

Keywords: hypertension, DASH, blood pressure, free-living environment

Nutrition and Metabolic Insights 2010:3 15-23

This article is available from http://www.la-press.com.

(c) the author(s), publisher and licensee Libertas Academica Ltd.

This is an open access article. Unrestricted non-commercial use is permitted provided the original work is properly cited. 


\section{Introduction}

Approximately 50 million individuals in the United States and approximately 1 billion worldwide are affected with hypertension which is defined as systolic blood pressure $\geq 140 \mathrm{mmHg}$ and/or diastolic blood pressure $\geq 90 \mathrm{mmHg} .{ }^{1}$ Blood pressure (BP) measures of 120-139/80-89 $\mathrm{mmHg}$ are defined as pre-hypertension and place individuals at increased risk for progression to hypertension. ${ }^{1}$ Hypertension and pre-hypertension typically have no additional symptoms and therefore these conditions may go undetected for years causing damage to the arteries and increasing the risk for coronary heart disease, heart attack, heart failure, stroke, and kidney failure. ${ }^{1}$ The current national recommendations for preventing and managing pre- and stage 1 hypertension focus on controllable lifestyle modifications including: reducing weight for overweight or obese individuals, ${ }^{2,3}$ decreasing dietary sodium, ${ }^{4-6}$ increasing physical activity, ${ }^{7,8}$ moderating alcohol consumption, ${ }^{9}$ and adopting the dietary approaches to stop hypertension (DASH) diet. ${ }^{4,5}$

The DASH diet was designed to be rich in potassium, calcium, magnesium, fiber, and protein and low in saturated and total fat and cholesterol. ${ }^{10}$ It was also designed to utilize commonly purchased and consumed food items that meet the standards of the DASH diet including all food groups. The diet emphasizes consumption of fruits, vegetables, nuts, whole grains, low-fat dairy foods, fish, poultry, lean meat, and other foods that are low in saturated and total fat. ${ }^{10}$ The initial DASH feeding trial, sponsored by the National Heart, Lung and Blood Institute (NHLBI), was conducted as a multicenter, clinical investigation where all meals were prepared and premeasured on the premises and dispensed to study participants. Participants consumed one meal 5-days a week on-site and the remaining meals were taken home to be consumed in the home environment. The study consisted of a three week run-in period and eight week intervention period. ${ }^{10}$ This intervention resulted in a significant reduction in systolic BP (SBP) and diastolic BP (DBP) in pre-hypertensive $(3.5 / 2.2 \mathrm{mmHg})$ and stage 1 hypertensive $(11.6 / 5.3 \mathrm{mmHg})$ individuals. $^{10}$ The success of this initial clinical trial led to the publication of the DASH eating plan pamphlet (DASH diet) that contains seven daily breakfast, lunch, dinner and snack menus for following the DASH diet. ${ }^{11}$
The DASH diet has subsequently been included as the current clinical guidelines for blood pressure control, ${ }^{1}$ and has been included in the recommendations by the Dietary Guidelines for Americans since 2005. ${ }^{12}$

In subsequent clinically controlled studies evaluating the DASH diet, all meals were again prepared and pre-measured on the premises and dispensed to study participants. ${ }^{13,14}$ In clinical investigations incorporating behavioral lifestyle interventions that focused on losing weight, increasing physical activity, reducing sodium intake, and limiting alcohol consumption, study participants were either counseled on the DASH diet ${ }^{15}$ or provided with all meals,${ }^{14}$ as previously described. These clinical investigations demonstrated greater reductions in SBP/DBP, 9.5/5.3 $\mathrm{mmHg}^{15}$ and 11.1/6.4 $\mathrm{mmHg},{ }^{14}$ in individuals following the DASH diet and corresponding behavioral modifications compared to control groups. Despite, the success of the clinical feeding trials $\mathrm{s}^{10,13}$ and subsequent clinical interventions combining the DASH diet with other behavioral modifications, ${ }^{14,15}$ the ability of adopting the original DASH diet followed in a free-living environment where no pre-measured meals are provided and without any additional behavioral modifications has only been evaluated in a single study. ${ }^{16}$ This previous study assessed the effect of an internet-based nutrition education program, which was based on the DASH diet, on body weight and blood pressure over 12 months. While this intervention resulted in significantly reduced body weight and blood pressure, only $26 \%$ of the participants who began the program, completed all 12 months. Furthermore, this intervention was limited to those individuals with access to an internet-computer and the understanding of how to utilize a web-based computer-site.

The purpose of this investigation was to provide descriptive data as to the blood pressure changes pre- and stage 1 hypertensive individuals following the DASH diet, in a free-living environment (e.g. at home), can expect versus a group of similar individual following no dietary advice (control). In this study participants received dietary advice in person and over the telephone. It was hypothesized that individuals following the DASH diet in a free-living environment would exhibit significantly reduced SBP and DBP relative to control group. Additionally, because changes in body weight, BMI, 
total kilocalories, ${ }^{2,3}$ and sodium intake ${ }^{4-6}$ have been identified as potential moderators of blood pressure, these variables were monitored during the 4-week intervention. The relationship between changes in these variables and changes in blood pressure over the 4-week intervention was then assessed. Finally, selfreported physical activity behavior was also assessed prior to and at the conclusion of the intervention.

\section{Methods}

\section{Participants}

Individuals were recruited for this investigation from The Kent State University campus and neighboring communities. Email and special project announcements were sent to all faculty, staff and students once a week for a three-week cycle. Initial screening was conducted through email correspondence by providing clarification of the specific eligibility requirements including age, medical history, blood pressure, and medication as well as the willingness to follow a diet modification for a period of 4 weeks. One hundred and sixteen volunteers were eliminated prior to commencement of screening. Twenty-four volunteers with either pre- or stage 1 hypertension who were not taking anti-hypertensive medications attended three screening visits at the Nutrition Laboratory at Kent State University. Resting blood pressure was assessed twice per screening visit for a total of six assessments. Inclusion criteria for the study required volunteers to be 22-60 years of age and present with an average SBP $120-160 \mathrm{mmHg}$ and/ or DBP $80-95 \mathrm{mmHg}$ based on six measurements while not taking anti-hypertensive medications. In addition to BP assessments, volunteers completed a health history questionnaire. Any participant indicating one or more of the following conditions was subsequently excluded from the study: poorly controlled diabetes mellitus; hyperlipidemia; a cardiovascular event within the previous six months; pregnancy or lactation; use of medications that may affect blood pressure; unwillingness to stop taking vitamin and mineral supplements or antacids containing magnesium or calcium; renal insufficiency; and an alcoholic-beverage intake of more than 14 drinks per week. Four volunteers were eliminated before assignment to the groups due to low blood pressure. Group assignment was made based on individuals blindly picking a group-name tag (i.e. DASH or control) from a jar. Each participant began their 4-week intervention period after meeting the screening criteria. A total of twenty participants received either instruction to follow the DASH diet (DASH) (males $\mathrm{N}=5$, age $33.8 \pm 10.4$ years, females $\mathrm{N}=5$, age $43.2 \pm 10.0$ years) or follow their normal diet (control) (males $\mathrm{N}=7$, age $40.1 \pm 10.5$ years, females $\mathrm{N}=3$, age $33.3 \pm 13.1$ years) for a period of four weeks. Written informed consent was obtained from all participants prior to participation. This study was approved by the Institutional Review Board at Kent State University.

\section{Procedures}

\section{Screening visit one}

During the first screening visit, study protocol and possible risks were explained. Informed consent was obtained and volunteers completed a health history questionnaire. Volunteers' height $(\mathrm{cm})$ and body weight $(\mathrm{kg})$ were assessed with a stadiometer (Perspective Enterprises: Kalamazoo, MI) and bariatric platform scale (Vogel, Halke, GmbH \& Co; Hamburg, Germany) respectively. BP was assessed twice with a random-zero sphygmomanometer (Am Diagnostic Corp, Hauppauge, NY) and stethoscope (Marshall Electronics, Inc. Skokie, IL) using standard BP assessment protocol. ${ }^{1}$ An appropriate-sized cuff (i.e. cuff bladder encircling at least $80 \%$ of the arm) was used to ensure accuracy for each participant. Upon leaving, volunteers were given forms and instructions to complete 3-day dietary intake records. ${ }^{17}$

\section{Screening visit two}

The second screening visit was conducted a minimum of one week, but not more than 10 days, after visit one. $\mathrm{BP}$ was assessed again using the identical methodology from visit one. Completed 3-day dietary intake records were collected and reviewed with volunteers. Physical activity recall questionnaires designed as an integral part of a health history questionnaire were completed by volunteers at this time. ${ }^{18}$

\section{Screening visit three}

The third screening visit was conducted a minimum of one week, but not more than 10 days, after visit two. During this final screening visit, BP was again assessed using identical techniques as visit one and two. The average systolic and diastolic BP from 
each of the six measurements obtained over the three screening visits was calculated. Twenty participants who had an average SBP between $120-160 \mathrm{mmHg}$ and/or DBP between $80-95 \mathrm{mmHg}$ were deemed eligible for the study and separated into either the DASH or control group as described above.

\section{Diet intervention}

Immediately after a participant was deemed eligible for this investigation, the 4-week intervention phase began. The DASH group received a copy of the document, "Your Guide to Lowering Your Blood Pressure with DASH". ${ }^{11}$ The DASH booklet contained a complete 7-day set of 2,100-kcal menus which were cycled four times during the 4 week intervention period. Each DASH group participant received instructions for following each daily menu plan with their target energy intake level (see Instruments - dietary intake record). Participants were instructed to follow each of the daily menus based on their target energy intake levels in an effort to maintain their current body weight. Each participant in the control group was instructed to follow their regular diet regimen with their target energy intake levels (see Instruments - dietary intake record) for the 4 week intervention period. All participants were provided with dietary intake record forms for logging intake and one set of 3-day dietary records were collected at the end of week 4.

During the intervention period, each participant recorded their daily intake of food on the dietary intake forms provided. The DASH group was instructed to record all food items consumed even items not on the menu plans. Although alcohol was not listed on the menus, participants were allowed to consume up to 2 servings of alcohol a day which was similar to the initial DASH feeding trial. ${ }^{19}$ Each participant was contacted by phone on a weekly basis and symptom and physical activity recall questionnaires were used for documenting conversations. Participants were encouraged to discuss any problems with the DASH menu plans, to describe any adverse affects from the diet, and to discuss any weight loss, change in physical activity, or reason for noncompliance with the DASH menus. Despite some unusual abdominal symptoms experienced by the DASH group during the first week of the trial, symptoms did not persist and participants were not hindered from following the DASH diet.
During week 4, participants reported to the Nutrition Laboratory on two occasions. During the first visit, BP was assessed twice with identical techniques used during screening. On the second visit, two BP measures and body weight were assessed; 3-day dietary intake records were collected; and physical activity recall questionnaires were completed.

All participants received a $\$ 100$ grocery store gift card that was used to partially offset the purchase of food items during the intervention phase.

\section{Instruments}

\section{Dietary intake record}

During screening visit one, each volunteer was provided with dietary record forms to be completed prior to their second visit to the laboratory. Each volunteer was to record all food and beverage items consumed including brand names and total quantities for a total of two weekdays and one weekend day. Dietary records were collected at screening visit two and the data from these records were reviewed and clarified with participants by trained dietary personnel. The dietary records were entered into the NutriBase IV Clinical Software (CyberSoft, Inc., ver. 4.5). Reports for each volunteer for each day were printed and averages were calculated for total energy intake over the 3-day period. These calculated averages were the target energy intake level used for each volunteer to aid in stabilizing body weight. For small groups the 3-day average food record can provide a $95 \%$ reliable estimate of energy intake within $10 \%$ of a 1 -year average. ${ }^{17}$

During week 4 each participant completed a second set of 3-day dietary records which were collected on the final day of the intervention period. Recording of the dietary records was the identical process used during screening. Dietary records were reviewed and clarified with participants by trained dietary personnel, entered into the NutriBase IV Clinical Software, and reports for each participant for each day were printed that contained consumption of macro- and micronutrients. Post-intervention nutrient data represents the average intake calculated from the 3-day dietary records during week 4 .

\section{Physical activity recall questionnaire}

Physical activity was controlled by monitoring changes in activity through physical activity recall 
questionnaires ${ }^{18}$ that were completed by participants during the screening period and the 4th week of the intervention. In addition, the dietitian completed the physical activity recall questionnaires during weekly phone conversions based on each participant's response. Participants were asked not to change their activity level (e.g. do not began a new exercise program) during the 4 week period. The physical activity recall questionnaires consisted of 5 questions that included 1) how much physical work is required at your job/school; 2) outside of work/school how often do you engage in exercise/physical activity/ sports; 3) what did you do including mode of activity, duration, frequency, and intensity; 4) how long have you engaged in this activity; and 5) list any other physical activity/exercise/sports performed during the week. Estimated energy expenditure was based on the selected physical activities that each participant stated on their physical activity recall questionnaire. The mode of activity and the intensity were used to determine the metabolic equivalent (METS) for each activity the participant performed at baseline and week $4 .{ }^{20}$ The estimated energy expenditure was calculated using the following formulas:

Energy expenditure $(\mathrm{kcal} /$ minute $)=[0.0175 \mathrm{kcal}$. $\mathrm{kg}^{-1} \cdot \mathrm{MET}^{-1} \times$ METS $\times$ body weight $\left.(\mathrm{kg})\right] \times$ duration (minutes) $\times$ frequency (\# of days/week).

The physical activity level assigned to each participant was based on the frequency of estimated energy expenditure per week. ${ }^{21}$ The classifications assigned included sedentary for $<1000 \mathrm{kcals} /$ week; light for 1000-1500 kcals/week; and moderate for $>1500 \mathrm{kcals} / \mathrm{week}$.

\section{Statistical analyses}

Independent sample T-tests were utilized to examine differences in participant height and age between groups (DASH, control). Two separate, two group (DASH, control) by two time (pre, post) analysis of variance (ANOVA) were performed to determine differences in both SBP and DBP. Multiple group by two time ANOVAs were used to determine differences in potential dietary (total kilocalories, sodium intake) and anthropometric (body weight, BMI) moderators of blood pressure. Post-hoc analysis for significant main and interaction effects from ANOVAs were performed using T-tests. Pearson's correlation analysis was then used to determine the relationship between changes in blood pressure (SBP, DBP) and changes in potential dietary (total kilocalories, sodium intake) and anthropometric (body weight, BMI) moderators of blood pressure over the 4-week intervention.

Chi-square analysis was performed to determine if differences existed between groups for the number of participants who reported sedentary, light, and moderate physical activity during baseline assessments. An additional Chi-square analysis was performed to determine if there was a difference in the number of participants in each group who indicated increases in their physical activity patterns over the course of the treatment.

Gender was initially included as an independent variable in the analysis of the effect of the DASH diet on blood pressure. However in this initial analysis there were no significant main or interaction effects of gender $(P>0.19)$. This suggests that in the present study, both males and females responded similarly to the treatment and control conditions. Therefore, gender was not included as an independent variable in the final analysis presented herein.

\section{Results}

Participants in the DASH and control groups were not significantly $(P \geq 0.7)$ different in age (DASH: $38.5 \pm 10.5$ years, and control: $38.1 \pm 11.1$ years) or height (DASH: $173.1 \pm 8.8 \mathrm{~cm}$, and control: $174.6 \pm 7.0 \mathrm{~cm})$. Two-way ANOVA demonstrated that there was a significant group (DASH, control) by time (pre, post) interaction for $\operatorname{SBP}(P=0.003)$ (Fig. 1). The interaction was due to a significant decrease $(P<0.001)$ in SBP in the DASH group (pre: $141.3 \pm 11.3 \mathrm{mmHg}$, post: $130.7 \pm 9.1 \mathrm{mmHg}$ ) over the course of the intervention with no change $(P=0.32)$ in SBP noted in the control group (pre: $133.5 \pm 6.6 \mathrm{mmHg}$, post: $131.9 \pm 8.9 \mathrm{mmHg}$ ). There was also a significant main effect for differences in SBP (pre: $137.4 \pm 9.9 \mathrm{mmHg}$, post: $131.3 \pm 8.7 \mathrm{mmHg}$ ) over time $(P<0.001)$, however this difference was driven by the reduction in SBP exhibited by the DASH group which is outlined in the interaction. There were no significant main or interaction effects for group or time $(P>0.17$ for both) for differences in DBP(DASH pre: $87.9 \pm 6.6 \mathrm{mmHg}$, post: $85.7 \pm 7.8 \mathrm{mmHg}$; control pre: $86.5 \pm 5.2 \mathrm{mmHg}$, post: $85.2 \pm 6.8 \mathrm{mmHg}$ ). 


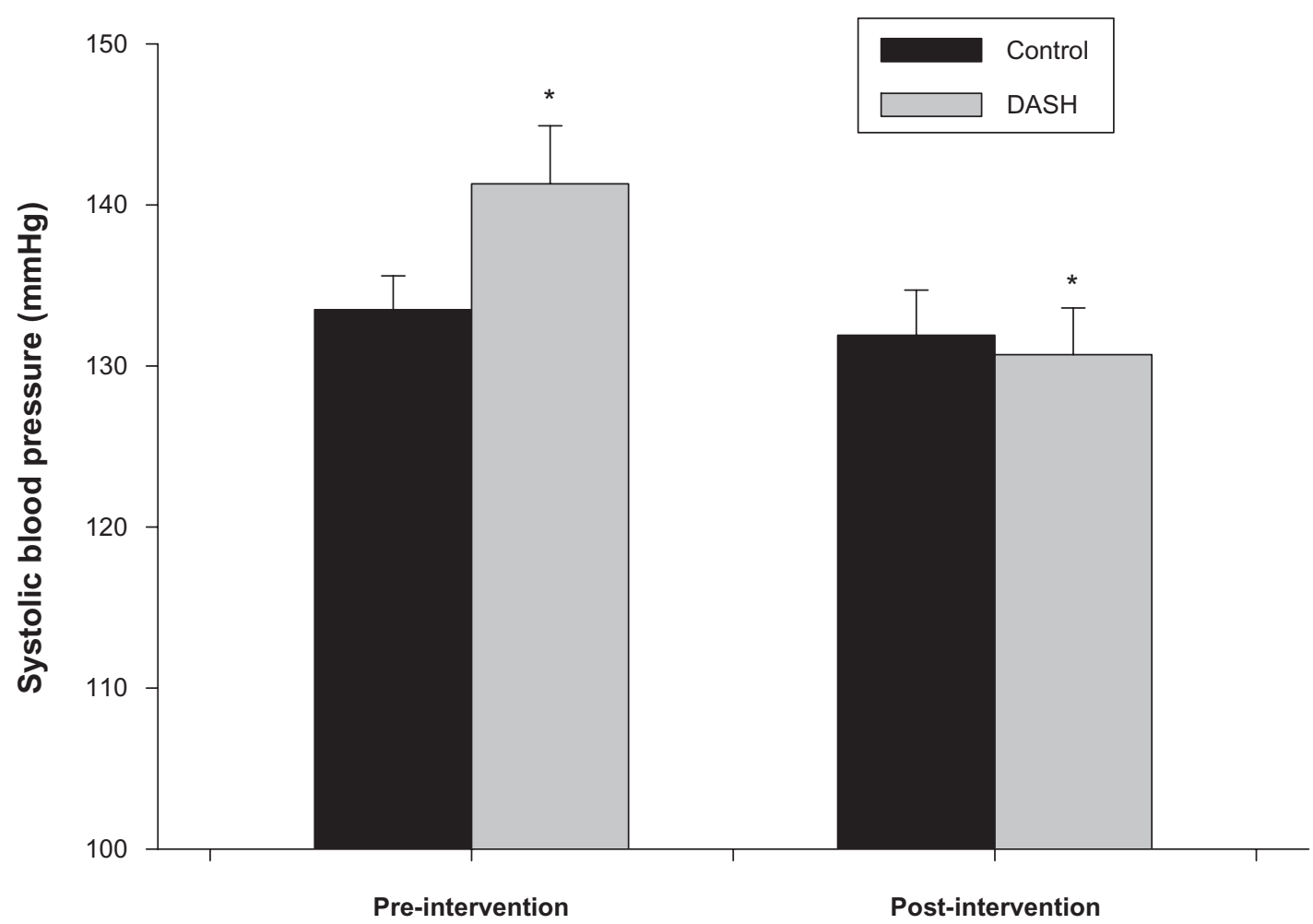

Figure 1. Systolic blood pressure pre and post-intervention in DASH and control groups. Significant group (DASH, control) by time (pre, post-intervention) interaction $(P=0.003)$. *Similar symbols were significantly different $(P<0.001)$.

Potential moderators of blood pressure are shown in Table 1. There were significant main effects of time $(P \leq 0.004)$ and group $(P \leq 0.008)$ for body weight and BMI. There was an overall reduction in body weight and BMI from pre- to post-treatment and participants in the DASH group had a significantly greater body weight and BMI than those in the control group. There was not a significant time by group interaction for differences in body weight or BMI $(P \geq 0.14)$. There was a significant main effect of time $(P \leq 0.001)$ for sodium intake and total kilocalories due to an overall reduction in sodium intake and total kilocalories pre- to post-treatment.
There were no significant main or interaction effects $(P \geq 0.14)$ of group for differences in sodium intake or total kilocalories. Pearson's correlation analyses revealed that changes in body weight, BMI, sodium intake, and total kilocalories were each not associated with changes in SBP $(\mathrm{r} \leq 0.14, P \geq 0.5)$ or DBP ( $\mathrm{r} \leq 0.10, P \geq 0.6$ ) pre- to post-treatment.

Before the intervention, five, three and two of the 10 DASH participants and one, three and six of the 10 control participants reported sedentary, light and moderate physical activity respectively. The number of participants who indicated sedentary $(P=0.10)$, light $(P=1.0)$ or moderate $(P=0.16)$ physical

Table 1. Potential moderators of blood pressure.

\begin{tabular}{|c|c|c|c|c|}
\hline & \multicolumn{2}{|c|}{ DASH $(N=10)$} & \multicolumn{2}{|c|}{ Control $(\mathbf{N}=10)$} \\
\hline & Pre & Post & Pre & Post \\
\hline Weight (kg) & $102.6 \pm 20.8$ & $100.7 \pm 20.6$ & $79.8 \pm 12.0$ & $79.2 \pm 11.6$ \\
\hline BMI & $34.5 \pm 8.2$ & $33.9 \pm 8.2$ & $26.1 \pm 2.9$ & $25.9 \pm 2.7$ \\
\hline *Total Kilocalories & $2900 \pm 1093$ & $1990 \pm 658$ & $2331 \pm 660$ & $1888 \pm 790$ \\
\hline *Sodium (mg) & $5288 \pm 2388$ & $3325 \pm 1576$ & $4036 \pm 1861$ & $3006 \pm 1245$ \\
\hline
\end{tabular}

Data are mean \pm SD.

${ }^{*}$ Significant main effects for time for total kilocalories and sodium intake ( $P \leq 0.001$ for both). 
activity was not different between the two groups when analyzed with Chi-square. After treatment four of the 10 DASH participants and one of the 10 control participants indicated an increase in physical activity during the intervention. Chi-square analysis demonstrated that the number of participants who indicated increased physical activity during the intervention was not different between groups $(P=0.18)$.

\section{Discussion}

Clinical feeding trials of the original ${ }^{10}$ and modified ${ }^{13,22}$ DASH diet and DASH diet in conjunction with other behavioral modifications ${ }^{14}$ have been conducted in controlled environments. One study has evaluated the DASH diet with additional behavioral modifications in a free-living environment, ${ }^{15}$ while another study has evaluated a modified version of the DASH diet in a free-living environment. ${ }^{23}$ This second study was conducted outside the United States and as such the participants' diets were different from that of a typical American diet. ${ }^{23}$ To the best of our knowledge there has only been a single study that previously evaluated the efficacy of the original DASH diet with no other behavioral modifications in a free-living environment on individuals who follow a typical American diet. ${ }^{16}$ That previous study utilized an internet-based instructional method whereas the present study uses a traditional person-to-person instructional technique. Presently, individuals following the DASH diet in a free-living environment exhibited a significantly greater reduction in SBP relative to controls; however, DBP was not significantly altered in either group. Because changes in body weight, BMI, sodium intake, and total kilocalories were not presently associated with changes in blood pressure and because each group did not differentially participate in physical activity, the reductions in SBP noted here appear to be independent of these variables.

Previously, clinical feeding evaluations of the DASH and modified DASH diets reported average reductions ranging from 5.5 to $9.5 \mathrm{mmHg}$ and 2.9 to $5.7 \mathrm{mmHg}$ in SBP and DBP respectively. ${ }^{10,13,14,22}$ Free-living evaluations of variations of the DASH diet or the DASH diet in combination with additional behavioral modifications have reported average reductions ranging from 1.8 to $11.1 \mathrm{mmHg}$ and 0.7 to $6.4 \mathrm{mmHg}$ in SBP and DBP respectively. ${ }^{15,23}$ Presently, the original DASH diet followed in a free-living environment in a group of pre- and stage 1 hypertensive individuals using a traditional personto-person instructional technique elicited reductions in SBP $(10.6 \mathrm{mmHg})$ and DBP $(2.2 \mathrm{mmHg})$ that were similar to these previous studies..$^{10,13,22}$

Changes in four potential moderators of blood pressure; body weight, BMI, sodium intake, and total kilocalories were also assessed in the present study. Presently there was an overall reduction (both groups combined) in each of these variables. While this was likely due to the dietary recommendations in the DASH group, the act of participating in a study which evaluated dietary habits and blood pressure may have influenced changes in these variables in the control group as well. ${ }^{24}$ Changes in these variables were not associated with changes in SBP or DBP. This is contrary to previous evidence that has outlined the impact these variables have in blood pressure changes..$^{2-6}$ It is unclear why these variables were not associated with changes in blood pressure in the present investigation. It is possible that our sample size was too small to detect a significant association. Because of the nature of correlation analysis, examining a greater number of participants likely would have resulted in greater correlation coefficients and perhaps significant associations between these moderators and blood pressure. It is also possible that it is a combination of changes in these factors (i.e. reductions in body weight with reductions in sodium intake) that would explain the efficacy of the DASH diet in the present investigation. Furthermore, our method of assessing sodium intake, while valid, was likely not as accurate as an objective measure. Future evaluations of sodium intake with the DASH diet should consider a more accurate measure such as 24-hour urinary sodium measurements. Finally, other potential moderators, such as changes in alcohol intake $\mathrm{e}^{9}$ may have impact on blood pressure changes and although used as eligibility criterion presently were not evaluated.

\section{Limitations}

While the present study demonstrates the significant blood pressure changes one could expect to see while following the DASH diet in a free-living environment with no additional behavioral modifications, there are some limitations to the study. The current sample size $(\mathrm{N}=20)$ was small and results can only be generalized to pre-or stage 1 hypertensive adults between 
22 to 60 years of age who are otherwise healthy. As mentioned previously this likely limited our ability to demonstrate significant relationships between changes in potential moderators of blood pressure and the observed changes in blood pressure. Despite the small sample size the reduction of resting SBP in the DASH group was large enough to achieve statistical significance with the present analysis. Furthermore, the mean reductions in SBP $(10.6 \mathrm{mmHg})$ and $\mathrm{DBP}(2.2 \mathrm{mmHg})$ in the DASH group in the present study were similar to those from the original large clinical feeding studies of pre- and stage 1 hypertensive individuals. ${ }^{10,13,22}$ Because the present reductions were similar to what was seen in previous feeding studies which employed very large sample sizes, it would appear to be unlikely that examining additional participants would have drastically altered the average blood pressure reductions noted with the DASH and control groups in the present study. However, analyzing additional participants may have provided statistical significance for reductions in DBP in the DASH group.

\section{Conclusions}

The purpose of this study was to provide descriptive data as to the changes in blood pressure individuals following the DASH diet in a free-living environment could expect to achieve. To the best of our knowledge this is only the second study to evaluate the efficacy of the original DASH diet with no other behavioral modifications in a free-living environment in the United States. ${ }^{16}$ This was the first to do so using a traditional person-to-person instructional technique. Participants following the DASH diet achieved a reduction in SBP of $10.6 \pm 6.7 \mathrm{mmHg}$, which was significant and a reduction in DBP of $2.2 \pm 5.9 \mathrm{mmHg}$, which was not significant. These reductions were very similar to previous clinical and free-living evaluations of the DASH diet. Presently, none of the potential moderators of blood pressure we evaluated were independently associated with the observed changes in blood pressure. This may be due to our small sample size or the possibility that it is the combined change in multiple factors that impact blood pressure. Future research on the effects of the DASH diet in a free living environment should consider a greater sample size, more accurate measures of sodium intake and/or evaluation of additional potential moderators such as alcohol intake.

\section{Acknowledgments}

This study was partially funded through the Kent State University Research and Graduate Studies Department.

\section{Disclosures}

This manuscript has been read and approved by all authors. This paper is unique and is not under consideration by any other publication and has not been published elsewhere. The authors and peer reviewers of this paper report no conflicts of interest. The authors confirm that they have permission to reproduce any copyrighted material.

\section{References}

1. Chobanian AV, Bakris GL, Black HR, et al. The seventh report of the Joint National Committee on prevention, detection, evaluation, and treatment of high blood pressure. Journal of the American Medical Association. 2003;289:2560-72.

2. The Trials of Hypertension Prevention Collaborative Research Group Effects of weight loss and sodium reduction intervention on blood pressure and hypertension incidence in overweight people with high-normal blood pressure. Archives of Internal Medicine. 1997;157:657-67.

3. He J, Whelton PK, Appel LJ, Charleston J, Klag MJ. Long-term effects of weight loss and dietary sodium reduction on incidence of hypertension. Hypertension. 2000;35:544-9.

4. Sacks FM, Svetkey LP, Vollmer WM, et al; for the DASH-Sodium Collaborative Research Group. Effects on blood pressure of reduced dietary sodium and the dietary approaches to stop hypertension (DASH) diet. New England Journal of Medicine. 2001;344:3-10.

5. Vollmer WM, Sacks FM, Ard J, et al; for the DASH-Sodium Trial Collaborative Research Group. Effects of diet and sodium intake on blood pressure: subgroup analysis of the DASH-sodium trial. Annals of Internal Medicine. 2001;135:1019-28

6. Chobanian AV, Hill M, National Heart, Lung, and Blood Institute workshop on sodium and blood pressure: a critical review of current scientific evidence. Hypertension. 2000;35:858-63.

7. Kelley GA, Kelley KS. Progressive resistance exercise and resting blood pressure. Hypertension. 2000;35:838-43.

8. Whelton SP, Chin A, Sin X, He J. Effect of aerobic exercise on blood pressure. Annals of Internal Medicine. 2002;136:493-503.

9. Xin X, He J, Frontini MG, et al. Effects of alcohol reduction on blood pressure. Hypertension. 2001;38:1112-7.

10. Sacks FM, Appel LJ, Moore TJ, et al. A dietary approach to prevent hypertension: a review of the dietary approaches to stop hypertension (DASH) study. Clinical Cardiology. 1999;22(Suppl III):III-6-10.

11. U.S. Department of Health and Human Services, National Heart, Lung, and Blood Institute. Your guide to lowering your blood pressure with DASH. Available at: http://www.nhlbi.nih.gov/health/public/heart/ hbp/dash. Accessed February 2009.

12. Executive of the President and the U.S. Department of Health and Human Services. Brief summary: dietary guidelines for Americans, 2005. Available at: http://www.healthierus.gov. Accessed February 2009.

13. Svetkey LP, Sacks FM, Obarzanek E, et al. The DASH diet, sodium intake and blood pressure trial (DASH-sodium): rationale and design. Journal of the American Dietetic Association. 1999;99(Suppl):S96-104.

14. Miller III ER, Erlinger TP, Young DR, et al. Results of the diet, exercise, and weight loss intervention trial (DEW-IT). Hypertension. 2002;40:612-8.

15. Svetkey LP, Harsha DW, Vollmer WM, et al. Premier: A clinical trial of comprehensive lifestyle modification for blood pressure control: rationale, design and baseline characteristics. Annals of Epidemiology. 2003;13:462-71. 
16. Moore JM, Alsabeeh N, Apovian CM, et al. Weight, blood pressure, and dietary benefits after 12 months of a web-based nutrition education program (DASH for health): longitudinal observational study. Journal of Medical Internet Research. 2008;10(4):e52.

17. Basiotis PP, Welsh SO, Cronin FJ, Kelsay JL, Mertz W. Number of days of food intake records required to estimate individual and group nutrient intakes with defined confidence. Journal of Nutrition. 1987;117:1638-41.

18. Nieman DC. Exercise Testing and Prescription: A Health-Related Approach 4th ed. Mountain View, CA: Mayfield Publishing Co;1999.

19. Sacks FM, Obarzanek E, Windhauser MM, et al. Rationale and design of the dietary approaches to stop hypertension trial (DASH): a multicenter controlled-feeding study of dietary patterns to lower blood pressure. Annals of Epidemiology. 1995;5:108-18.

20. Ainsworth BE, Haskell WL, Leon AS, et al. Compendium of physical activities: classification of energy cost of human physical activities. Medicine and Science in Sports and Exercise. 1993;25:71-80.
21. American College of Sports Medicine (ACSM). ACSM's guidelines for exercise testing and prescription 8th ed. Baltimore: Lippincott Williams \& Wilkins; 2009:152-82.

22. Appel LJ, Sacks FM, Carey VJ, et al. Effects of protein, monounsaturated fat and carbohydrate intake on blood pressure and serum lipids: results of the Omni Heart randomized trial. Journal of the American Medical Association. 2005;294:2455-64

23. Nowson CA, Worsley A, Margerison C, et al. Blood pressure response to dietary modifications in free-living individuals. Journal of Nutrition. 2004; $134: 2322-9$

24. Writing Group of the Premier Collaborative Research Group. Effects of comprehensive lifestyle modification on blood pressure control: main results of the Premier Clinical Trial. Journal of the American Medical Association. 2003;289:2083-93.

\section{Publish with Libertas Academica and every scientist working in your field can read your article}

"I would like to say that this is the most author-friendly editing process I have experienced in over 150 publications. Thank you most sincerely."

"The communication between your staff and me has been terrific. Whenever progress is made with the manuscript, I receive notice. Quite honestly, l've never had such complete communication with a journal."

" $L A$ is different, and hopefully represents a kind of scientific publication machinery that removes the hurdles from free flow of scientific thought."

Your paper will be:

- $\quad$ Available to your entire community free of charge

- $\quad$ Fairly and quickly peer reviewed

- Yours! You retain copyright

http://www.la-press.com 